\title{
Perfil sociodemográfico e clínico do paciente com doença crônica atendido em um serviço de emergência
}

\author{
Sociodemographic and clinical profile of the patient with chronic disease treated at an emergency \\ service
}

Perfil sociodemográfico y clínico del paciente con enfermedad crónica atendido en un servicio de urgências

\author{
Denise Ferro \\ ORCID: https://orcid.org/0000-0002-7025-9080 \\ Universidade de São Paulo, Brasil \\ E-mail: denise.ferro@usp.br \\ Luciana Aparecida Fabriz \\ ORCID: https://orcid.org/0000-0001-7633-0127 \\ Universidade Estadual do Oeste do Paraná, Brasil \\ E-mail: luciana.fabriz@gmail.com \\ Tatiele Estefâni Schönholzer \\ ORCID: https://orcid.org/0000-0002-4294-8807 \\ Faculdade FATEC de Ivaiporã, Brasil \\ E-mail: tatischonholzer@gmail.com \\ Carolina Grigolato Viola \\ ORCID: https://orcid.org/0000-0003-1950-5538 \\ Universidade de São Paulo, Brasil \\ E-mail: carolina.grigolato.viola@usp.br \\ Silvia Helena Valente \\ ORCID: https://orcid.org/0000-0002-3593-9590 \\ Universidade de São Paulo, Brasil \\ E-mail: silviavalente@usp.br \\ Fabaina Costa Machado Zacharias \\ ORCID: https://orcid.org/0000-0003-1150-6114 \\ Universidade de São Paulo, Brasil \\ E-mail: fabiana.zacharias@usp.br \\ Ione Carvalho Pinto \\ ORCID: https://orcid.org/0000-0001-7541-5591 \\ Universidade de São Paulo, Brasil \\ E-mail: ionecarv@eerp.usp.br
}

\begin{abstract}
Resumo
Objetivo: Identificar as características sociodemográficas e clínicas de usuários com Doenças Crônicas Não Transmissíveis (DCNT) atendidos em um serviço de emergência hospitalar. Metodologia: Trata-se de uma pesquisa transversal quantitativa, realizada no prontuário eletrônico de 364 pacientes diagnosticados em pelo menos um dos quatro principais grupos de DCNT, conforme a Classificação Internacional de Doenças (CID-10), em um serviço de emergência de um hospital público de alta complexidade localizado no noroeste do Estado de São Paulo. Utilizou-se estatística descritiva para análise dos dados. Resultados: Houve predominância de pacientes do sexo masculino $(53,3 \%)$, casados $(56,0 \%)$, com ensino fundamental incompleto $(49,2 \%)$ com idade média de 62,7 anos. O diagnóstico com maior predominância $(77,7 \%)$ foram as Doenças Cardiovasculares (DCV). As comorbidades associadas com maior frequência entre os participantes do estudo foram Hipertensão Arterial Sistêmica (HAS) (31,9\%), seguido de tabagismo (22,3\%), dislipidemia (19,2\%) e etilismo (16,5\%). Dos participantes do estudo, 80,4\% possuíam complicações relacionadas ao seu diagnóstico principal, sendo a principal complicação Acidente Vascular Cerebral (AVC) prévio (18,4\%). O medicamento mais utilizado para tratamento entre os participantes foi a classe de antihipertensivos. Além disso, encontraram-se extensivos erros nos registros dos prontuários clínicos dos usuários, acarretando em subnotificações. Conclusão: Conhecer o perfil dos usuários que frequentam serviço de emergência hospitalar torna-se fundamental considerando que se trata, muitas vezes, da agudização de DCNT, as quais poderiam ser minimizadas caso houvesse um acompanhamento mais eficaz do usuário na Atenção Primária à Saúde (APS), reduzindo internações não planejadas, bem como gastos e superlotação dos serviços de emergência.
\end{abstract}

Palavras-chave: Perfil de saúde; Doença crônica; Serviços médicos de emergência. 


\begin{abstract}
Objective: To identify the sociodemographic and clinical characteristics of users with Chronic Noncommunicable Diseases (NCDs) treated at a hospital emergency service. Methodology: This is a quantitative cross-sectional survey, carried out on the electronic medical record of 364 patients diagnosed in at least one of the four main groups of NCDs, according to the International Classification of Diseases (ICD), in an emergency service of a hospital highly complex public located in the northwest of the State of São Paulo. Descriptive statistics were used for data analysis. Results: There was a predominance of male patients (53.3\%), married (56.0\%), with incomplete elementary education $(49.2 \%)$ with an average age of 62.7 years. The most prevalent diagnosis $(77.7 \%)$ was cardiovascular diseases (CVDs). The comorbidities most frequently associated among study participants were Systemic Arterial Hypertension (SAH) (31.9\%), followed by smoking (22.3\%), dyslipidemia (19.2\%) and alcoholism (16.5\%). Of the study participants, $80.4 \%$ had complications related to their main diagnosis, the main complication being previous stroke $(18.4 \%)$. The most used medication for treatment among the participants was the class of antihypertensive drugs. In addition, extensive errors were found in the records of users' medical records, resulting in underreporting. Conclusion: Knowing the profile of users who attend hospital emergency services becomes essential considering that it is often the aggravation of NCDs, which could be minimized if there was a more effective monitoring of the user in Primary Healthcare, reducing unplanned hospitalizations, as well as spending and overcrowding of emergency services.
\end{abstract}

Keywords: Health profile; Chronic disease; Emergency medical services.

\title{
Resumen
}

Objetivo: Identificar las características sociodemográficas y clínicas de los usuarios con Enfermedades Crónicas No Transmisibles (ENT) atendidos en un servicio de urgencias hospitalario. Metodología: se trata de una investigación cuantitativa transversal, realizada sobre la historia clínica electrónica de 364 pacientes diagnosticados en al menos uno de los cuatro principales grupos de ENT, según la Clasificación Internacional de Enfermedades (CIE-10), en un servicio de urgência en hospital público de alta complejidad ubicado en el noroeste del Estado de São Paulo. Se utilizó estadística descriptiva para el análisis de datos. Resultados: Predominó el sexo masculino (53,3\%), casados (56,0\%), con educación primaria incompleta $(49,2 \%)$, con una edad promedio de 62,7 años. El diagnóstico más prevalente $(77,7 \%)$ fue Enfermedades Cardiovasculares (ECV). Las comorbilidades asociadas más frecuentes entre los participantes del estudio fueron la hipertensión arterial sistémica (HAS) (31,9\%), seguida del tabaquismo (22,3\%), la dislipidemia (19,2\%) y el alcoholismo (16,5\%). De los participantes del estudio, el 80,4\% tuvo complicaciones relacionadas con su diagnóstico principal, siendo la principal complicación un acidente cerebrovascular previo $(18,4 \%)$. La medicación más utilizada para el tratamiento entre los participantes fue la clase de antihipertensivos. Además, se encontraron grandes errores en las historias clinicas de los usuarios, lo que resultó en un subregistro. Conclusión: Conocer el perfil de los usuarios que acuden a los servicios de urgencias hospitalarias se torna fundamental teniendo en cuenta que muchas veces se trata del agravamiento de las ENT, lo que podría minimizarse si hubiera un seguimiento más efectivo del usuario en Atención Primaria de Salud (APS), reduciendo las hospitalizaciones no planificadas, así como el gasto y el hacinamiento de los servicios públicos.

Palabras-clave: Perfil de salud; Enfermedad crónica; Servicios médicos de urgência.

\section{Introdução}

A inversão da pirâmide populacional mundial, com diminuição das taxas de natalidade e o aumento do contingente de pessoas idosas, acarreta em elevação exacerbada das doenças crônicas não-transmissíveis (DCNT), constituindo estas, como um problema de saúde pública. Elas compõem as principais causas de morbimortalidade mundial, com $71 \%$ da taxa de óbitos globais, 15 milhões de mortes prematuras (indivíduos entre 30 a 70 anos) (OMS, 2018). Além disso, demandam a maior parte dos atendimentos em saúde, com gastos estimados de US\$ 7 trilhões durante o período de 2011-2025 em países de baixa e média renda (Aquino et al., 2012, OMS, 2018).

As DCNT englobam diabetes mellitus (DM); doenças cardiovasculares (DCV); cânceres; doenças respiratórias crônicas; doenças infecciosas persistentes; condições ligadas à maternidade e ao período perinatal; condições ligadas à manutenção da saúde por ciclos de vida; distúrbios mentais de longo prazo; deficiências físicas e estruturais contínuas; doenças metabólicas e doenças bucais (Mendes, 2012).

Estudos analisaram dados da Pesquisa Nacional de Saúde (PNS) realizada em 2013 e mostraram que 54 milhões de indivíduos (cerca de $45 \%$ da população adulta brasileira) relatam pelo menos uma DCNT, sendo mais prevalentes: a hipertensão arterial sistêmica (HAS) (21,4\%), depressão (7,6\%), artrite (6,4\%) e DM (6,2\%) (Malta et al., 2015, Theme Filha 
et al., 2015).

Logo, ao avaliar sua cronicidade, a gravidade das complicações e os meios necessários para controle das DCNT, compreende-se que estas deixam de ser apenas um recorte da etiopatogênia e passam a ser a forma como se estrutura a resposta social do sistema de saúde aos problemas e complexidades que estas requerem, tendo em vista que a condição crônica afeta indivíduo, família e sociedade. Diante desse contexto, em 2011, o Ministério da Saúde (MS) lançou o Plano de Ações Estratégicas para o Enfrentamento das DCNT 2011-2022, o qual estabeleceu metas e ações necessárias para a redução das taxas de mortalidade prematura por essas doenças em pelo menos, $2 \%$ ao ano, bem como a diminuição da prevalência de seus fatores de risco associados às mesmas (Brasil, 2011).

Historicamente e, mesmo com a criação da Política Nacional de Atenção às Urgências e Emergências, instituída em 2011 pelo MS, ainda nos dias atuais o sistema de saúde brasileiro responde às demandas de doenças crônicas de forma fragmentada, episódica e reativa, e se voltam principalmente para as condições agudas de saúde e a agudização das DCNT. (Dias et al., 2018).

Na maioria das vezes, o atendimento pontual se dá nos serviços de emergência, os quais acabam por atender casos clínicos não urgentes e funcionam como "portas de entrada" do Sistema Único de Saúde (SUS), 24 horas por dia, gerando vaga zero, pacientes nos corredores, tempo de espera para atendimento elevado, além de estresse e incertezas na equipe assistencial (Santos et al., 2014). Além disso, o uso frequente de serviços de emergência por usuários de DCNT pode ser entendido como um evento sentinela de que o cuidado não está adequado nos demais pontos da rede (Coleman et al., 2015).

Diante da magnitude imposta pelo crescimento das DCNT no cenário nacional, bem como pela prioridade dada às unidades de urgência e emergência na gestão em saúde do governo, na interferência da avaliação que usuários, trabalhadores e sociedade fazem do direito à saúde, da assistência ofertada e da integralidade do cuidado, faz-se necessário conhecer o perfil do doente crônico que utiliza este serviço, de forma a adequá-lo para que o mesmo possa suprir as necessidades do usuário e o encaminhá-lo para seguimento na Rede de Atenção à Saúde (RAS). E, portanto, este estudo objetivou identificar as características sociodemográficas e clínicas de usuários com doenças crônicas, que passaram por atendimento em um serviço de emergência hospitalar.

\section{Metodologia}

Este estudo, extraído dos resultados de tese de doutorado em finalização, caracteriza-se por uma pesquisa quantitativa, descritiva e transversal (Vieira \& Hossne, 2015), realizada em um hospital público de alta complexidade localizado noroeste do Estado de São Paulo. O hospital conta com 167 leitos de internação e possui uma equipe multiprofissional (médicos, enfermagem, fisioterapia, terapia ocupacional, psicologia, dentre outros), com atendimento 24 horas por dia. Além disso, realiza cerca de 2500 atendimentos mensais de urgência referenciada, que geram cerca de 900 internações e 450 cirurgias de alta complexidade mensais.

A população do estudo foi identificada a partir de relatórios de alta semanais gerados do sistema informatizado do hospital. Para o cálculo amostral, utilizou-se uma margem de erro de 5\%, nível de confiança de 95\% e uma estimativa de que 250 pacientes com DCNT recebiam alta semanalmente do serviço, estabelecendo-se uma amostra aleatória de 364 participantes $(n=364)$, diagnosticados em pelo menos um dos quatro principais grupos de condições crônicas (DM, DCV, doenças respiratórias e câncer), conforme Classificação Internacional de Doenças (CID-10) (Quadro 1), no momento de sua admissão no hospital, entre os meses de dezembro de 2018 a junho de 2019. Após o recebimento da lista, foi realizada uma busca no prontuário eletrônico de cada paciente com objetivo de identificar os potenciais participantes para o estudo, considerando os seguintes critérios de inclusão: possuir pelo menos um CID-10 incluso no Quadro 1, ter 18 anos ou mais e ser 
residente do município de Ribeirão Preto.

Quadro 1 - Lista de códigos referentes aos diagnósticos incluídos no estudo, conforme Classificação Internacional de Doenças, Ribeirão Preto, 2019.

\begin{tabular}{|lc|}
\hline \multicolumn{1}{|c|}{ Doenças } & Código (CID-10) \\
Câncer & $\mathrm{C} 00-\mathrm{C} 97$ \\
Neoplasias Malignas & $\mathrm{D} 00-\mathrm{D} 09$ \\
Neoplasias in situ & $\mathrm{E} 10-\mathrm{E} 14$ \\
Diabetes Mellitus & \\
Doenças Cardiovasculares & $\mathrm{I} 05-\mathrm{I} 09$ \\
Doença reumática crônica do coração & $\mathrm{I} 10-\mathrm{I} 15$ \\
Doenças hipertensivas & $\mathrm{I} 20-\mathrm{I} 25$ \\
Doenças isquêmicas do coração & $\mathrm{I} 60-\mathrm{I} 69$ \\
Doenças cerebrovasculares & $\mathrm{I} 26-\mathrm{I} 89$ \\
Outras doenças cardiovasculares & $\mathrm{J} 40-\mathrm{J} 44$ \\
Doenças Respiratórias & $\mathrm{J} 45-\mathrm{J} 46$ \\
Doença pulmonar obstrutiva crônica & \\
Asma & \\
\hline
\end{tabular}

Fonte: Autores (2019).

A coleta de dados foi realizada a partir de um roteiro, o qual contemplou dados dos usuários com doenças crônicas, tais como sexo, idade, bairro de procedência em Ribeirão Preto, grau de instrução, CID-10 relacionado a atual internação, diagnóstico da doença, medicamentos utilizados para o tratamento, complicações relacionadas a patologia, comorbidades relacionadas, tempo de permanência no serviço e número de atendimentos no serviço no último ano, número de atendimentos na Atenção Primária à Saúde (APS) no último ano, reinternações no serviço em dois meses após alta, óbito em dois meses após alta. Os dados foram obtidos através dos prontuários eletrônicos, dispostos no sistema online do hospital, e posteriormente contempladas por ligação telefônica, caso os pesquisadores sentissem necessidade.

Os dados coletados foram transpostos para planilha no programa Microsoft Office Excel 2016, passando por dupla digitação independente. Foi aplicado análise descritiva dos dados com elaboração de tabelas de frequência absoluta (n) e percentual (\%) das variáveis categóricas e cálculo de medidas de posição e dispersão (média, mediana, desvio de padrão, valores mínimo e máximo) para as variáveis contínuas.

O estudo foi aprovado pelo Comitê de Ética em Pesquisa com Seres Humanos da Escola de Enfermagem de Ribeirão Preto da Universidade de São Paulo, atendendo a Resolução CNS n466/2012, Protocolo CAAE: 96780518.5.0000.5393.

\section{Resultados}

O estudo contou com 364 participantes, com predominância do sexo masculino $(53,3 \%)$, casados $(56,0 \%)$, de baixa escolaridade, com apenas o ensino fundamental incompleto (49,2\%), moradores da zona norte do município (44,5\%) e com idade média de 62,7 anos, conforme Tabela 1. 
Tabela 1 - Características sociodemográficas dos pacientes com doenças crônicas que receberam atendimento em um serviço de emergência, Ribeirão Preto, 2019.

\begin{tabular}{lc}
\hline Variáveis & $\mathrm{n}=364$ \\
\hline Sexo * & $194(53,3)$ \\
Masculino & $170(46,7)$ \\
Feminino & $62,7 \pm 15,2$ \\
Idade (anos) ${ }^{\dagger}$ & \\
Estado Civil * & $60(16,5)$ \\
Solteiro(a) & $204(56,0)$ \\
Casado(a) / União Estável / União Consensual & $45(12,4)$ \\
Divorciado(a) / Separado(a) & $55(15,1)$ \\
Viúvo(a) & \\
Grau de Escolaridade * & $30(8,2)$ \\
Analfabeto(a) & $179(49,2)$ \\
Ensino Fundamental Incompleto & $35(9,6)$ \\
Ensino Fundamental Completo & $14(3,8)$ \\
Ensino Médio Incompleto & $73(20,1)$ \\
Ensino Médio Completo & $9(2,5)$ \\
Ensino Superior Incompleto & $19(5,2)$ \\
Ensino Superior Completo & $5(1,4)$ \\
Pós-graduação (Mestrado, Doutorado, Especialização, etc.) & \\
Moradia por Distrito * & $162(44,5)$ \\
Norte & $30(8,2)$ \\
Sul & $49(13,5)$ \\
Leste & $114(31,3)$ \\
Oeste & $7(1,9)$ \\
Centro & \\
\hline
\end{tabular}

*Variáveis categóricas expressas por $\mathrm{n}(\%)$;

†Variável contínua expressa como média \pm desvio padrão.

Fonte: Autores (2019).

Entre os participantes, houve predominância como diagnóstico principal de CID-10 do grupo DCV (77,7\%), sendo que $80,4 \%$ dos participantes do estudo possuíam complicações relacionadas ao seu diagnóstico principal. Além disso, 327 participantes possuíam comorbidades associadas, conforme Tabela 2.

Tabela 2 - Características clínicas dos pacientes com doenças crônicas que receberam atendimento em um serviço de emergência, Ribeirão Preto, 2019.

\begin{tabular}{lc}
\hline Variáveis & $\mathrm{n}=364$ \\
\hline Doença crônica (por grupo de causa) * $^{*}$ Câncer & $91(25)$ \\
$\quad$ Diabetes Mellitus & $101(27,7)$ \\
$\quad$ Doenças Cardiovasculares & $283(77,7)$ \\
$\quad$ Doenças Respiratórias & $33(9,1)$ \\
Comorbidade associada * \# & $327(92,4)$ \\
Complicações * \# & $283(80,4)$ \\
Uso de medicamentos * \# $_{\text {Tempo de permanência no serviço de emergência (dias) }{ }^{\dagger}}^{\dagger}$ & $320(92,5)$ \\
Número de atendimentos no serviço de emergência em um ano ${ }^{\dagger}$ & $4(1-60)$ \\
Reinternação no serviço de emergência no período de dois meses após alta ${ }^{\dagger}$ & $1(1-7)$ \\
Óbito no período de dois meses após alta * $^{\dagger}$ & $0(0-5)$ \\
Frequentam Unidade Básica de Saúde * & $25(6,9)$ \\
Número de atendimentos na Unidade Básica de Saúde em um ano ${ }^{\dagger}$ & $304(83,5)$ \\
\hline
\end{tabular}

*Variáveis categóricas expressas por $\mathrm{n}(\%)$;

\#Utilizada porcentagem válida devido a omissão de dados após coleta de dados;

†Variável assimétricas expressas como mediana e intervalo interquartil.

Fonte: Autores (2019). 
É importante ressaltar que o município do estudo possui três grandes hospitais de referência para urgências e emergências, sendo o referenciamento feito pela Central Única de Regulação Médica (CURM), que avalia os pedidos de internação e transferência hospitalar, estabelecendo prioridades e o fluxo de pacientes. Portanto, há uma grande possibilidade do número de reinternações, bem como, o número de óbitos após dois meses da alta dos participantes expostos na Tabela 2, seja subnotificado neste estudo, tendo em vista que não há quaisquer garantias da reinternação (caso seja necessária) ser feita no hospital no qual o paciente esteve internado anteriormente.

Além disso, o serviço de emergência onde foi realizado o presente estudo opera, na grande maioria das vezes, em vaga zero e consequente superlotação, o que poderia acarretar em uma grande possibilidade do referenciamento do usuário ser feito para um dos outros dois hospitais destinados ao mesmo atendimento e, também, como o prontuário eletrônico do paciente não é integrado na rede, sendo que, cada serviço possui seu próprio sistema de informação, não há a possibilidade de saber com precisão se houve ou não internações prévias ou reinternações dos participantes nestes hospitais - houve a tentativa de questionar os participantes por contato telefônico à respeito de internações prévias nos outros dois hospitais, porém, as informações não eram precisas, optando-se apenas, por considerar, para este estudo, o que estava registrado no sistema do presente hospital.

Neste estudo, observou-se que 275 participantes possuíam CID-10 secundários. O diagnóstico (tanto principal como secundário) com maior prevalência dentre os participantes do estudo foi o I10 (Hipertensão Essencial - Primária) com 34,1\%, seguido de I64 (Acidente Vascular Cerebral, não especificado como hemorrágico ou isquêmico) com 16,5\%, I67-8 (Outras doenças cerebrovasculares especificadas) com 14,0\%, E11-9 (Diabetes mellitus não-insulinodependente - sem complicações) com 11,8\% e I48 (Flutter e fibrilação atrial) com 10,4\%, conforme Tabela 3.

Durante a coleta de dados do estudo, notou-se a existência de um preenchimento inadequado por parte da equipe médica do serviço de emergência em relação aos diagnósticos por CID-10 (primários e secundários), no prontuário eletrônico, durante atendimento realizado ao paciente, resultando em subnotificação dos diagnósticos que levaram os usuários a internação no serviço.

Tabela 3 - Distribuição dos diagnósticos principais e secundários, por CID-10, recebidos com maior frequência entre os pacientes com doenças crônicas que receberam atendimento em um serviço de emergência, Ribeirão Preto, 2019.

\begin{tabular}{lc}
\hline Diagnóstico & $\mathrm{n}(\%)$ \\
\hline I10 - Hipertensão Essencial - Primária & $124(34,1)$ \\
I64 - Acidente Vascular Cerebral, não especificado como hemorrágico ou isquêmico & $60(16,5)$ \\
I67-8 - Outras doenças cerebrovasculares especificadas & $51(14,0)$ \\
E11-9 - Diabetes mellitus não-insulinodependente - sem complicações & $43(11,8)$ \\
I48 - Flutter e fibrilação atrial & $38(10,4)$ \\
I11-0 - Doença cardíaca hipertensiva com insuficiência cardíaca (congestiva) & $32(8,8)$ \\
I25-1 - Doença aterosclerótica do coração & $29(8,0)$ \\
E11-2 - Diabetes mellitus não-insulino-dependente - com complicações renais & $27(7,4)$ \\
I12-0 - Doença renal hipertensiva com insuficiência renal & $22(6,0)$ \\
I13-2 - Doença cardíaca e renal hipertensiva com insuficiência cardíaca (congestiva) e & \\
insuficiência renal & $20(5,5)$ \\
I20-0 - Angina instável & $20(5,5)$ \\
I21-9 - Infarto agudo do miocárdio não especificado & $18(4,9)$ \\
E14-9 - Diabetes mellitus não especificado - sem complicações & $16(4,4)$ \\
\hline
\end{tabular}

Fonte: Autores (2019).

Assim, diante do preenchimento inadequado em relação aos diagnósticos principais e secundários que acarretaram em atendimento hospitalar durante internação, apresentou-se nas comorbidades associadas, da mesma forma que nos diagnósticos 
(principais e secundário) por CID-10, uma maior prevalência de participantes hipertensos (31,9\%), seguido dos tabagistas $(22,3 \%)$, dislipidêmicos $(19,2 \%)$ e etilistas $(16,5 \%)$, conforme Tabela 4.

Tabela 4 - Distribuição das comorbidades associadas com maior frequência entre pacientes com doenças crônicas que receberam atendimento em um serviço de emergência, Ribeirão Preto, 2019

\begin{tabular}{lc}
\hline Comorbidades Associadas & $\mathrm{n}(\%)$ \\
\hline Hipertensão Arterial Sistêmica & $116(31,9)$ \\
Tabagista & $81(22,3)$ \\
Dislipidemia & $70(19,2)$ \\
Etilista & $60(16,5)$ \\
Ex-tabagista & $51(14,0)$ \\
Obesidade & $36(9,9)$ \\
Diabetes Mellitus & $29(8,0)$ \\
Doença Renal Crônica & $29(8,0)$ \\
Ex-etilista & $28(7,7)$ \\
Hipotiroidismo & $27(7,4)$ \\
Depressão & $24(6,6)$ \\
Doença Arterial Obstrutiva Periférica & $20(5,5)$ \\
Hiperplasia Prostática Benigna & $17(4,7)$ \\
Cirrose Hepática & $17(4,7)$ \\
Epilepsia & $15(4,1)$ \\
Úlcera Gastrointestinal & $12(3,3)$ \\
Doença Arterial Coronariana & $10(2,7)$ \\
\hline
\end{tabular}

Fonte: Autores (2019).

As principais complicações apresentadas pelos participantes do estudo foram Acidente Vascular Cerebral (AVC) prévio (18,4\%), metástase (14,3\%), Infarto Agudo do Miocárdio (IAM) prévio (11,3\%), paresia hemiparesia/paraparesia/tetraparesia $(9,1 \%)$, cateterismo cardíaco $(7,4 \%)$, angioplastia cardíaca $(6,3 \%)$ e nefropatia diabética (5,5\%), seguidos de disartria $(4,9 \%)$, implantação de stent no coração $(4,1 \%)$, recidiva oncológica $(3,8 \%)$, amputação de membro (3,3\%), uso de Sonda Nasoentérica (SNE) ou Sonda Nasogástrica (SNG) (3,0\%) e neuropatia diabética (2,7\%).

O medicamento mais utilizado para tratamento entre os participantes deste estudo foi o Ácido Acetilsalić́lico (AAS), com 133 (36,5\%) participantes fazendo uso; seguido pelo uso de Sinvastatina para 104 (28,6\%) participantes; e Omeprazol em 89 casos $(24,5 \%)$. Além disso, enalapril foi utilizado por 84 (23,1\%) destes participantes; 83 participantes (22,8\%) faziam uso de losartana, 74 participantes $(20,3 \%)$ faziam uso de furosemida. Observou-se que anti-hipertensivos são a classe de medicamentos mais utilizados dentre os participantes deste estudo.

Ressalta-se que, considerando as complicações relacionadas à patologia e medicações utilizadas para o tratamento, notou-se que também há um registro inadequado de todas as informações histórico clínico de alguns participantes. Ainda, em relação ao uso de medicamentos, na maioria das vezes, inclusive, o próprio participante não sabia referir o nome dos medicamentos que utilizava, durante contato telefônico. Diante disso, como exposto na Tabela 2, para obter a frequência também se utilizou uma porcentagem válida devido à ausência destes dados, durante todo o processo de obtenção dos dados.

\section{Discussão}

Considerando o objetivo deste estudo que foi o de identificar as características sociodemográficas e clínicas de usuários com doenças crônicas, que passaram por atendimento em um serviço de emergência encontrou-se que a maioria dos usuários do referido serviço eram homens, casados, de baixa escolaridade (ensino fundamental incompleto), moradores da zona norte do município e com idade média de 62,7 anos, achados que corroboram com outros estudos (Anversa, 2020). 
A utilização intensa dos serviços de emergências é determinada pela necessidade percebida pelo usuário e resulta principalmente da situação de saúde e do conhecimento prévio de sua DCNT. No contexto brasileiro, a doença é a responsável direta pelo consumo destes serviços de saúde (Malta et al., 2017).

Estudos demonstram que as mulheres possuem maior percepção quanto aos sinais e sintomas das doenças e, consequentemente, maior procura dos serviços na APS, praticando a promoção e prevenção de doenças (Malta et al., 2017, Simieli et al., 2019). Quando se trata da vulnerabilidade e altas taxas de morbimortalidade, percebe-se que os homens não buscam atendimento na APS e adentram ao sistema de saúde por meio de serviços de emergência e, caso houvesse uma maior a procura da APS por estes, diversos agravos e internações em serviços de emergência poderiam ser evitados (Mello et al., 2017).

Ainda, em relação ao sexo dos usuários, estudos demonstram que os homens são mais vulneráveis às doenças, em especial aquelas graves e crônicas, ocupando mais leitos em Unidades de Terapia Intensiva (UTI) e vindo a óbito mais rapidamente do que as mulheres (Bidinotto et al. 2016, Simão et al., 2019).

Um estudo demonstra que doentes crônicos com baixa escolaridade possuem maior prevalência de internação e de abandono de atividades por motivo de saúde, bem como, maior prevalência de passar por consultas médicas, o que corrobora com outros estudos realizados que indicam maior prevalência de DCNT em populações menos escolarizadas (Malta et al., 2017, Acosta et al., 2020)

Estudo recente demonstra que há maior prevalência de DCNT entre usuários do serviço de saúde do sexo masculino que residem em bairros de baixa renda, e conclui que, faz-se necessário considerar toda a realidade complexa envolta das favelas, sendo marcada pela desigualdade social, condições precárias de saneamento, habitações insalubres, bem como a falta de serviços de saúde suficientes para atendimento à toda população (Melo et al., 2019). Diante disso, é unânime para os pesquisadores que, para melhorar a saúde e, consequente diminuição da mortalidade nesta população vulnerável, é necessário que os gestores planejem intervenções que abordem estes importantes determinantes sociais em saúde (Barreto et al., 2017).

Ao se considerar as características clínicas dos participantes do estudo, houve predominância, como diagnósticos principal e secundário, de doenças do grupo DCV, sendo que o diagnóstico com maior prevalência dentre os participantes do estudo foi o I10 (Hipertensão Essencial - Primária) com 34,1\%, sendo que, a HAS também foi a principal comorbidade associada entre a maioria deles. Este achado corrobora com estudos que trazem a crise hipertensiva como um dos principais motivos para busca aos serviços de emergência (Acosta, 2015; Lima et al., 2019)

A principal complicação apresentada pelos participantes deste estudo foi AVC, sendo que, 18,4\% já haviam sofrido AVC previamente. Um estudo apontou que um dos principais fatores de risco para que ocorra um novo AVC é justamente a HAS (78,4\%), bem como o AVC prévio (28,8\%) (Barella et al., 2019). Além disso, observou-se que, seguindo uma lógica, os anti-hipertensivos foram a classe de medicamentos mais utilizados dentre os participantes, sendo que o baixo nível de controle da HAS, bem como sua agudização é, na maioria das vezes, reflexo de uma adesão inadequada ao tratamento anti-hipertensivo. Assim, um estudo evidenciou que a quantidade de vezes em que o paciente foi admitido serviço de emergência nos últimos dois anos era influenciável pela adesão à terapia medicamentosa, ou seja, aqueles pacientes que não procuraram o serviço, ou o fizeram apenas uma vez, tiveram melhor adesão ao uso de anti-hipertensivos quando comparados àqueles que tiveram maior frequência de admissões no serviço de emergência (Albuquerque et al., 2018).

Dessa forma, observa-se que 304 (83,5\%) participantes do estudo referem fazer acompanhamento de suas DCNT em Unidades Básicas de Saúde (UBS), com uma mediana de 2 consultas no último ano. Um estudo com enfoque em internações por Condições Sensíveis à Atenção Primária (CSAP) no Brasil, constatou que em 11 lugares onde a Estratégia Saúde da Família (ESF) foi presente, os índices de hospitalização em serviços de emergência por DCNT eram 13\% abaixo daquelas onde a ESF tinha baixa participação (Mello et al., 2017). 
Além disso, corrobora com outro estudo que aponta a presença de um perfil de pacientes em um serviço de emergência que poderiam ser atendidos por UBS ressaltando as fragilidades do acesso e do cuidado continuado na APS, de forma a demonstrar a importância do trabalho da atenção básica, proporcionando um melhor desempenho ao SUS, por meio da diminuição de internações consideravelmente evitáveis (Lima et al., 2019).

Considera-se como uma importante limitação do estudo a ausência de preenchimento dos diagnósticos na maioria dos prontuários dos usuários pela equipe médica do serviço de emergência, resultando em uma subnotificação importante das DCNT, impedindo os gestores de conhecer a real magnitude, bem como poder gerir de forma adequada os recursos disponíveis para o enfrentamento destas. Além disso, outra limitação importante deste estudo é a ausência de um sistema de informação interligado entre os serviços de emergência o que proporciona a perda de informações importantes a respeito da continuidade do cuidado, número de reinternações e óbitos subsequentes no município.

\section{Conclusão}

Considerando o objetivo deste estudo que foi o de identificar as características sociodemográficas e clínicas de usuários com doenças crônicas, que passaram por atendimento em um serviço de emergência hospitalar, conclui-se que, determinadas causas de internações nos serviços de emergência como, por exemplo, a agudização de DCNT, principalmente a HAS, poderiam ser minimizadas, caso houvesse um acompanhamento mais eficaz do usuário na APS, pois acredita-se que o seguimento na atenção básica é capaz de diminuir internações por complicações da doença, como crises hipertensivas, doenças cerebrovasculares e insuficiência cardíaca congestiva, bem como sequelas resultantes destas complicações. Além disso, acarretaria em diminuição dos gastos públicos com o setor, a superlotação dos serviços, proporcionando um atendimento de emergência eficaz e de qualidade.

Ainda, conhecendo o perfil sociodemográfico e clínico dos usuários que frequentam serviços de emergência, esperase contribuir para investigações futuras que visem propostas de intervenção, educação e melhoria, possibilitando potencializar as políticas públicas de saúde relacionadas a estes serviços.

\section{Referências}

Acosta, A. M. \& Lima, M. A. D. S. (2015). Frequent users of emergency services: associated factors and reasons for seeking care. Revista Latino-Americana de Enfermagem, 23(2), 337-344. https://dx.doi.org/10.1590/0104-1169.0072.2560

Acosta, A. M., Lima, M. A. D. S., Pinto, I. C. \& Weber, L. A. F. (2020). Transição do cuidado de pacientes com doenças crônicas na alta da emergência para o domicílio. Revista Gaúcha de Enfermagem, 41(spe), e20190155. Epub 30 de abril de 2020. https://dx.doi.org/10.1590/1983-1447.2020.20190155

Albuquerque, N. L. S., Oliveira, A. S. S., Silva, J. M. \& Araújo, T. L. (2018). Associação entre acompanhamento em serviços de saúde e adesão terapêutica anti-hipertensiva. Revista Brasileira de Enfermagem, 71(6), 3006-3012. https://doi.org/10.1590/0034-7167-2018-0087

Anversa, A. C., Ponte, A. S., Machado, M. A. \& Fedosse, E. (2020). Avaliação do cuidado às condições crônicas de saúde: a perspectiva do usuário. Research, Society and Development, 9(4), e01942777. https://doi.org/10.33448/rsd-v9i4.2777

Aquino, E. M., Barreto, S. M., Bensenor, I. M., Carvalho, M. S., Chor, D., Duncan, B. B. \& et al (2012). Brazilian Longitudinal Study of Adult Health (ELSABrasil): objectives and design. Am J Epidemiol, 175(4), 315-24. https://doi.org/10.1093/aje/kwr294

Barella, R., Duran, V. de A. A., Pires, A. \& Duarte, R. (2019). Perfil do atendimento de pacientes com Acidente Vascular Cerebral em um hospital filantrópico do sul de Santa Catarina e estudo de viabilidade para implantação da unidade de AVC. Arquivos Catarinenses de Medicina, 48(1), 131-143. http://www.acm.org.br/acm/seer/index.php/arquivos/article/view/432

Barreto, M. L. (2017). Desigualdades em Saúde: uma perspectiva global. Ciência \& Saúde Coletiva, 22(7), 2097-2108. http://doi.org/10.1590/141381232017227.02742017

Bidinotto, D. N. P. B., Simonetti, J. P. \& Bocchi, S. C. M. (2016). A saúde do homem: doenças crônicas não transmissíveis e vulnerabilidade social. Revista Latino-Americana de Enfermagem, 24(spe), e2756. http://doi.org/10.1590/1518-8345.0735.2756

Brasil, Ministério da Saúde (2011). Secretaria de Vigilância em Saúde. Departamento de Análise de Situação de Saúde. Plano de ações estratégicas para o enfrentamento das doenças crônicas não transmissíveis (DCNT) no Brasil 2011-2022. Brasília: Ministério da Saúde. https://bvsms.saude.gov.br/bvs/publicacoes/plano_acoes_enfrent_dent_2011.pdf 
Research, Society and Development, v. 10, n. 7, e12010716263, 2021

(CC BY 4.0) | ISSN 2525-3409 | DOI: http://dx.doi.org/10.33448/rsd-v10i7.16263

CID-10. Classificação Estatística Internacional de Doenças e Problemas Relacionados com a Saúde. https://www.cid10.com.br/

Coleman, E. A. \& Roman, S. P. (2015). Family caregivers' experiences during transitions out of hospital. Journal for Healthcare Quality, Skokie, 37(1), 12-21. http://doi.org/10.1097/01.JHQ.0000460117.83437.b3

Dias, S. R. S., Santos, L. L. L. \& Silva, I. A. (2018). Classificação de risco no serviço de urgência e emergência: revisão integrativa da literatura. Rev Enferm UFPI., 7(1), 57-62. https://doi.org/10.26694/2238-7234.7157-62

Malta, D. C., Stopa, S. R., Szwarcwald, C. L., Gomes, N. L., Silva Júnior, J. B. \& Reis, A. A. C. dos. (2015). A vigilância e o monitoramento das principais doenças crônicas não transmissíveis no Brasil - Pesquisa Nacional de Saúde, 2013. Revista Brasileira de Epidemiologia, 18(Suppl. 2), 3-16. https://dx.doi.org/10.1590/1980-5497201500060002

Malta, D. C., Bernal, R. T. I., Lima, M. G., Araújo, S. S. C. de, Silva, M. M. A. da, Freitas, M. I. de F. \& et al. (2017). Noncommunicable diseases and the use of health services: analysis of the National Health Survey in Brazil. Revista de Saúde Pública, 51(Suppl. 1), 4s. Epub June 01, 2017. https://dx.doi.org/10.1590/s1518-8787.2017051000090

Melo, S. P. da S. de C., Cesse, E. A. P., Lira, P. I. C., Rissin, A., Cruz, R. de S. B. L. C. \& Batista Filho, M. (2019). Doenças crônicas não transmissíveis e fatores associados em adultos numa área urbana de pobreza do nordeste brasileiro. Ciência \& Saúde Coletiva, 24(8), 3159-3168. Epub August 05, 2019. https://dx.doi.org/10.1590/1413-81232018248.30742017

Mello, J. M., Borges, P. K. de O., Muller, E. V., Grden, C. R. B., Pinheiro, F. K. \& Borges, W. S. (2017). Hospitalizations for ambulatory care sensitive noncommunicable diseases of the circulatory system. Texto \& Contexto - Enfermagem, 26(1), e3390015. Epub April 13, 2017. https://dx.doi.org/10.1590/0104-07072017003390015

Mendes, E. V. (2012). O cuidado das condições crônicas na atenção primária à saúde: o imperativo da consolidação da estratégia da saúde da família. Brasília: Organização Pan-Americana da Saúde, 512 p.

Lima, A. C. M. G., Nichiata, L. Y. I. \& Bonfim, Daiana. (2019). Perfil dos atendimentos por condições sensíveis à Atenção Primária à Saúde em uma Unidade de Pronto Atendimento. Revista da Escola de Enfermagem da USP, 53, e03414. Epub January 31, 2019. https://doi.org/10.1590/s1980-220x2017042103414

Organização Mundial da Saúde (2018). Relatório da Comissão Independente Mundial de Alto Nível da OMS sobre Doenças Crônicas Não Transmissíveis (DCNTs): Time to Deliver. OMS.

Oliveira, A. D. da S., Sousa, F. da S., Martins, I. M., Cardoso, S. de B., Rocha, F. C. V. \& Cordeiro, E. C. O. (2014). Análise das causas e consequências da superlotação dos serviços de emergências hospitalares: uma revisão bibliográfica. Rev Saúde e Desen., 5(3), 31-44. https://doi.org/10.26694/2238-7234.724145

Simão, L., Lages, L., de Paiva, M., Ribeiro, N., Araújo, E. \& Leão, G. (2019). Perfil dos idosos com Doenças Crônicas Não Transmissíveis internados em Unidade de Terapia Intensiva. Enfermagem em Foco, 10(1). https://doi.org/10.21675/2357-707X.2019.v10.n1.1329

Simieli, I., Padilha, L. A. R. \& Tavares, C. F. de F. (2019). Realidade do envelhecimento populacional frente às doenças crônicas não transmissíveis. Revista Eletrônica Acervo Saúde, (37), e1511. https://doi.org/10.25248/reas.e1511.2019

Theme Filha, M. M., Souza Junior, P. R. B. de, Damacena, G. N. \& Szwarcwald, C. L. (2015). Prevalência de doenças crônicas não transmissíveis e associação com autoavaliação de saúde: Pesquisa Nacional de Saúde, 2013. Revista Brasileira de Epidemiologia, 18(Suppl. 2), 83-96. https://dx.doi.org/10.1590/1980-5497201500060008

Vieira, S. \& Hossne, W. S. (2015). Metodologia Científica para a Área da Saúde. $2^{\mathrm{a}}$ ed. Rio de Janeiro: Editora Elsevier. 